\title{
BIAS VARIATION BETWEEN TWO CO-LOCATED GNSS RECEIVERS
}

\author{
M. P. Olaniya*, S. Yadav, P. Kandpal, S. Panja, and A. Agarwal \\ CSIR-National Physical Laboratory, Dr. K. S. Krishnan Marg, New Delhi - 110012 \\ *olaniyamp@nplindia.org
}

Time and Frequency Metrology Section of CSIR-National Physical Laboratory, India has two dual frequency GNSS timing receivers. The receivers are being used for establishing primary traceability link to SI second for realizing local Universal Coordinated Time UTC(NPLI). The GNSS based navigation timing solution are based on pseudorange measurement made by correlating user receiver generated replica signals with the signals broadcast by the GNSS satellites. Biases arise in the receiver during the correlation process remain common and uniform across all receiver channels and eventually that common bias will cancelled out in the user navigation solution but will appear as fixed bias for timing solution. The timing bias includes the internal delays within the GNSS receiver and antenna hardware accounting the combined effect of the multi frequency and also the offset of the local timing reference i.e. UTC(NPLI). The reference $10 \mathrm{MHz}$ and 1PPS to these receivers is the steered output of Active Hydrogen Maser (AHM). Earlier the reference was high performance Cs-clock and the link was using two single frequency receivers. The study has done for long period and found that the bias variation between these dual frequency receivers exist with time. Although these receivers are placed in environment controlled room but outside environment is varying. The antenna and antenna cable are affected with the outside environment. The critical parameter of the environment are temperature and humidity which are varying $0^{0}$ to $50^{\circ} \mathrm{C}$ and 0 to $100 \%$ respectively over the year. The peak to peak variation between these receivers is observed $\sim 2$ ns using P3 analysis. The receivers used in this analysis are PolaRx3eTR (make Septentrio) and TTS-4 (make PikTime). The bias between two co-located GNSS Receivers is shown in the Figure 1. 


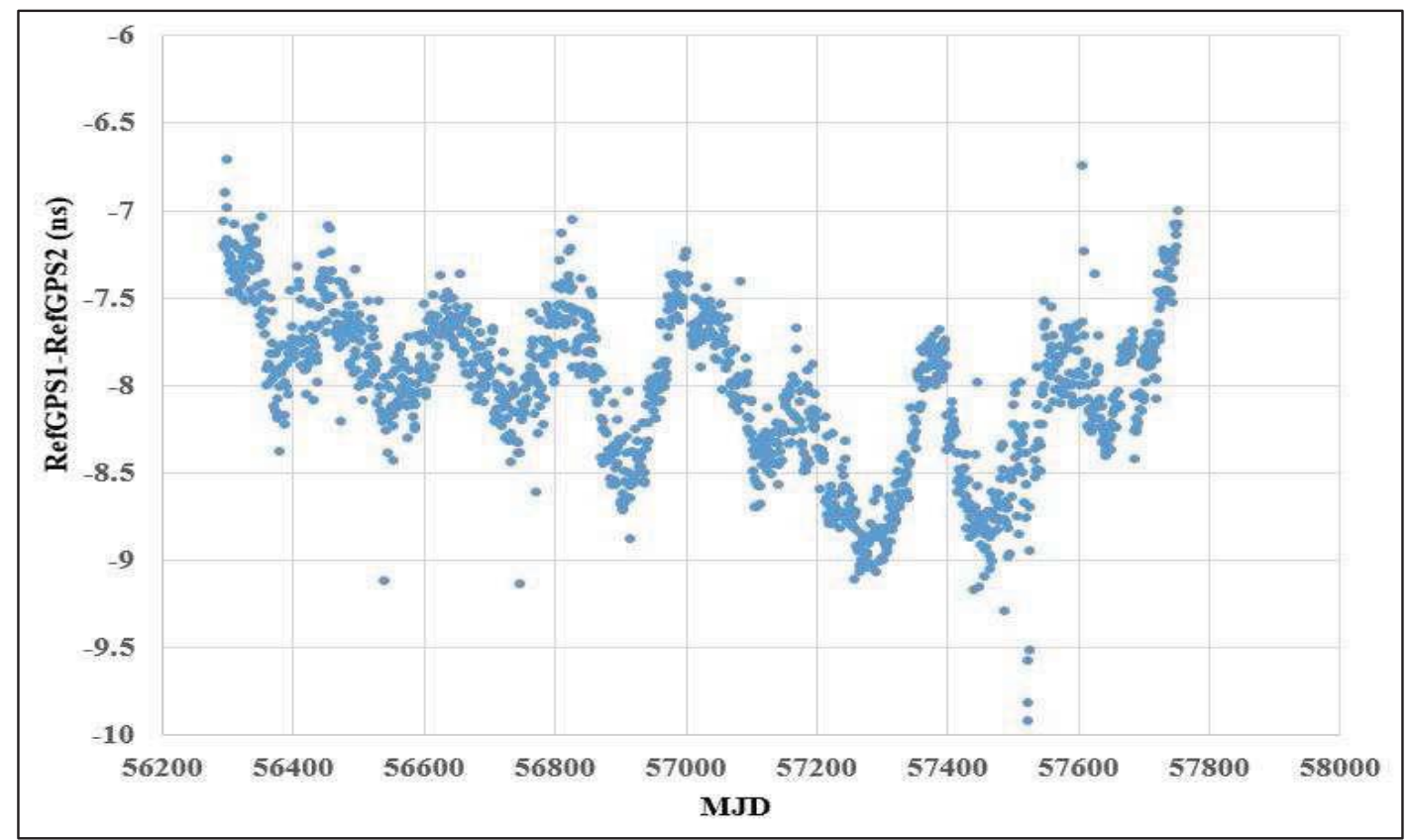

Figure1: Bias between two co-located GNSS Receivers from $1^{\text {st }}$ January, 2013 to $31^{\text {st }}$ December, 2016. RefGPS1 and RefGPS2 stands for TTS4 Rx and PolaRx3eTR Rx respectively. 